\author{
MITSUBISHI ELECTRIC RESEARCH LABORATORIES \\ http://www.merl.com
}

\title{
Efficient Medium Access Protocol for Wireless Sensor Networks
}

\author{
Jianlin Guo, Chunjie Duan, Ghulam Bhatti, Jinyun Zhang
}

TR2008-101 September 2008

\begin{abstract}
Efficiency of the medium access protocol is crucial for wireless sensor networks. For sensor nodes that are battery powered, it is sometimes difficulty or impractical to charge or replace exhausted battery. The sensor nodes collaborate for a common application such as environmental monitoring. The low sensing ranges result in dense networks. The medium access protocol (MAC) for wireless sensor network must be energy efficient. In this paper, we propose a timeslot sharing medium access protocol for wirless sensor networks. The proposed medium access protocol allows multiple sensor nodes to share a reserved or guaranteed timeslot for transmission. It also allows more sensor nodes to transmit in the reserved or guaranteed timeslots and fewer sensor nodes to contend for the transmission. The primary goal of timeslot sharing medium access protocol is to minimize the energy consumption of sensor nodes as well as to maximize the total number of nodes allowed in a network.
\end{abstract}

Sensorcomm 2008

This work may not be copied or reproduced in whole or in part for any commercial purpose. Permission to copy in whole or in part without payment of fee is granted for nonprofit educational and research purposes provided that all such whole or partial copies include the following: a notice that such copying is by permission of Mitsubishi Electric Research Laboratories, Inc.; an acknowledgment of the authors and individual contributions to the work; and all applicable portions of the copyright notice. Copying, reproduction, or republishing for any other purpose shall require a license with payment of fee to Mitsubishi Electric Research Laboratories, Inc. All rights reserved. 



\title{
Efficient Medium Access Protocol for Wireless Sensor Networks
}

\author{
Jianlin Guo, Chunjie Duan, Ghulam Bhatti, Jinyun Zhang \\ Mitsubishi Electric Research Laboratories \\ \{guo,duan,gbhatti,jzhang\}@merl.com
}

\begin{abstract}
Efficiency of the medium access protocol is crucial for wireless sensor networks. For sensor nodes that are battery powered, it is sometimes difficulty or impractical to charge or replace exhausted battery. The sensor nodes collaborate for a common application such as environmental monitoring. The low sensing ranges result in dense networks. The medium access protocol (MAC) for wireless sensor network must be energy efficient. In this paper, we propose a timeslot sharing medium access protocol for wireless sensor networks. The proposed medium access protocol allows multiple sensor nodes to share a reserved or guaranteed timeslot for transmission. It also allows more sensor nodes to transmit in the reserved or guaranteed timeslots and fewer sensor nodes to contend for the transmission. The primary goal of timeslot sharing medium access protocol is to minimize the energy consumption of sensor nodes as well as to maximize the total number of nodes allowed in a network.
\end{abstract}

\section{Introduction}

In a wireless sensor network, all sensor nodes share the same medium to transmit or receive packets. The collision, overhearing, control packet overhead, idle listening, and overemitting are the major reasons of energy waste [1]. It is necessary to enforce a medium access methodology in order to efficiently utilize the wireless medium and to minimize each node's energy consumption. A medium access protocol determines when and how sensor nodes can access the shared medium.

For synchronization and network management, wireless communication networks can partition time into periodic intervals, which are commonly called superframes $[2,3,4]$. A superframe can be further partitioned into a beacon period (BP), a data period
(DP), and in some cases, an idle period [2]. The data period can be further partitioned into a contention free period (CFP), and a contention access period (CAP). Figure 1 illustrates a superframe structure.

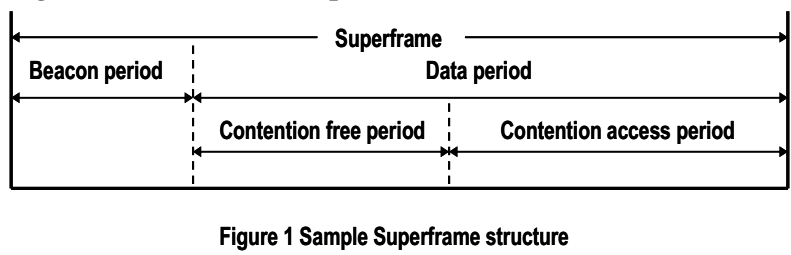

Each period consists of number of timeslots. The numbers of timeslots and the length of each timeslot in $\mathrm{BP}, \mathrm{CFP}$ and CAP can vary depending on specific network requirements.

During the BP, network control parameters are transmitted, i.e., network identifiers, transmission rates, logical channels, start of the CFP, the start of the CAP, the access schedule for the CFP, etc. The BP may consist of multiple timeslots [3] or a single timeslot [4]. During the CFP, sensor nodes reserve or are allocated timeslots to transmit data. During the CAP, sensor nodes use the contention technique such as Carrier Sense Multiple Access/Collision Detection $(\mathrm{CSMA} / \mathrm{CD})$ to transmit their packets.

In conventional medium access protocols, a timeslot in the BP or CFP is allocated to a specific node. In the case of the BP, the timeslot may be referred as a beacon slot. In the case of the CFP, the timeslot may be referred to as a reserved or guaranteed timeslot. During a timeslot in BP or CFP, only the node that has been allocated to the timeslot is allowed to transmit beacon or data. Even if the duration of the transmission is only a fraction of the timeslot or there is no transmission, other nodes are not allowed to use that timeslot unless the timeslot is reassigned to it. The timeslots in CAP are not allocated to any particular node. All nodes in the network are allowed to transmit in CAP by using the contention access mechanism defined for the given network. 
The superframe has a finite length and therefore the total numbers of timeslots in a superframe are limited. In practice, the superframe length is often limited. For example, the maximum number of the guaranteed timeslots in [2] is seven and [3] specifies a maximum of forty-eight beacon slots.

These conventional medium access protocols impose the following limitations on the wireless networks: The allocated timeslot is not fully utilized when the node does not have enough packets to transmit, which decreases the network efficiency. The destination node(s) need to stay awake for receiving in the reserved timeslots even if source node does not transmit any packet, and that results in idle listening. The total number of nodes that are allowed to transmit during the CFP is limited by the number of timeslots in CFP, which in a network with large number of nodes, forces more nodes contend for medium access during CAP, which in turn results in decrease of bandwidth efficiency due to the higher probability of collision. The retransmission caused by collisions also increases the energy consumption. In the extreme cases, it can cause the network outage. In some networks, all nodes are required to transmit their own beacon and listen to other beacons in every superframe [3]. Unnecessary beacon transmission increases the control packet overhead. Consequently, the conventional medium access protocols do not fully explore the characteristics of the wireless sensor networks. To achieve improved energy efficiency in medium access protocols, several medium access protocols specifically designed for wireless sensor networks are described in [1]. In this paper, we propose a timeslot sharing medium access protocol for wireless sensor networks to overcome the limitations imposed in conventional medium access protocols. The proposed timeslot sharing is both energy and bandwidth efficient and is a medium access protocol suitable for wireless sensor networks.

The remainder of the paper is structured as follows. In Section 2 we describe the timeslot sharing in the beacon period. The timeslot sharing in the contention free period is presented in Section 3. Paper is concluded in Section 4.

\section{Timeslot Sharing in Beacon Period}

The beacon is a special type of control packet transmitted by a given nodes. In some sensor networks, all nodes are required to transmit their beacon in a superframe [3]. The period where beacons are transmitted is defined as beacon period. Allowing a node to transmit beacon at a reduced frequency reduces the control packet overhead. Beacon slot sharing decreases the number of beacon slots and enables more time for data transmission, and therefore increases the efficiency of the network. Beacon slot sharing also allows nodes to save energy used to listen to and process unnecessary beacons. The principle of beacon slot allocation is to make use of beacon slot as efficient as possible and leave more time for data transmission.

In a controlled or managed wireless sensor network, one or more nodes are designated as network coordinator(s). The network coordinators are responsible for network management and resource allocation. Beacon transmission nodes request beacon slots from network coordinators during the process of network setup or joining network by sending their beacon transmission patterns to the network coordinators, which allocate beacon slots to beacon transmission nodes according to the beacon transmission needs of the requesting nodes. The network coordinators can allocate a single beacon slot to multiple beacon transmission nodes if there is no beacon collision. The network coordinators inform each of the nodes that share a beacon slot when and how to transmit beacon. Figure 2 shows an example of beacon slot sharing. Node A and node B share a beacon slot with node A transmitting beacon every four superframes and node B transmitting beacon every two superframes. Beacon transmission nodes are required to send their new beacon transmission patterns to the network coordinators when their beacon transmission patterns change. The network coordinators can change beacon slot allocation if it is necessary.



Figure 2 Node A and node B share beacon slot 3

In a controlled or managed wireless sensor network, the network coordinators can maintain a record of the beacon transmission patterns and beacon slot allocation for all beacon transmission nodes. A node can change its beacon transmission pattern and request new beacon slot allocation at any time. The network coordinators can also dynamically change the beacon slot allocation to maximize overall network efficiency. 
In a distributed wireless sensor network without network coordinator, beacon transmission nodes share the responsibility to manage beacon slot allocation. A beacon slot is said to be occupied if one or more nodes transmit beacons in that beacon slot. A node that transmits beacon announces its beacon transmission pattern in its beacon. A beacon transmission node first scans for an occupied beacon slot for sharing by listening to other nodes' beacons. If there is no occupied beacon slot satisfying its need it then selects an unoccupied beacon slot. To share an occupied beacon slot, a node needs to scan for beacon transmission in the shared beacon slot for a sufficient number of successive superframes to determine when it transmits beacon. For example, if node A selects an unoccupied beacon slot $\# 10$ and transmits beacon every two superframes. Node B that also transmits beacon every two superframes can share beacon slot \#10 with node A. To locate its turn for beacon transmission, node $\mathrm{B}$ has to monitor beacon transmission in beacon slot $\# 10$ for at least two consecutive superframes.

In such a distributed network, the beacon transmission nodes can detect beacon collision by skip its beacon transmission periodically. If a node that skips beacon transmission receives beacon in its beacon slot when it is its turn to transmit beacon, a beacon collision occurs. The node has to resolve the beacon collision by selecting a new beacon slot or new beacon transmission turn.

In a distributed wireless sensor network, a node can also change its beacon transmission pattern or beacon slot selection at any time and re-select or join a desirable beacon slot to transmit its beacon. A node can also change its beacon slot if it reduces the number of the occupied beacon slots or the length of beacon period, as long as a desirable beacon slot is available.

In both controlled and distributed wireless sensor networks, it should be noticed that nodes with different beacon transmission pattern or irregular beacon transmission pattern can also share a beacon slot as long as there is no beacon collision.

\section{Timeslot Sharing in Contention free Period}

The timeslot sharing in CFP is very important for dense wireless sensor networks. Generally, the sensor nodes do not have a large amount of data to transmit and are sleeping for most of time. A sensor node wakes up, performs its task, and goes to sleep again. A sensor node transmits its data in either an assigned timeslot in the CFP or in the CAP. Transmission in CFP allows a source node to wake up in the guaranteed timeslots and transmit data without contending for medium access. It also allows the destination node(s) to wake up in the exact timeslots and receive data without idle listening. In conventional MAC designs, once a timeslot is assigned to a node, it cannot be used by others regardless if the node has data to transmit.

A node that uses the CAP for data transmission only needs to wake up if it has data to transmit. However, in general, transmission in CAP consumes more energy compared to CFP transmission due to two factors: (a) The random backoff forces both transmitting nodes and receiving nodes to stay awake for a longer period of time; (b) Collision occurs for CAP transmission, which results in retransmissions.

Therefore, to achieve high energy-efficiency in dense wireless sensor networks, more sensor nodes should transmit in CFP and fewer sensor nodes should transmit in CAP. On the other hand, however, the number of timeslots in CFP is limited by the superframe period. Superframe length in a given systems is often chosen to achieve optimal system synchronization and extending the superframe length generally causes other issues and will result the diminishing of system efficiency.

The CFP timeslot sharing is designed to allow more nodes to utilize the limited slots in CFP in a sensor network. In a controlled or managed wireless sensor network, a node can request the guaranteed timeslots in CFP from a network coordinator to transmit data. The network coordinator can request a node to report its data transmission pattern (or bandwidth requirement) to determine timeslot allocation scheme. The network coordinator may allocate the sharable timeslots to a node as long as there is no transmission collision. The network coordinator informs a node about timeslot sharing information and node's turn to transmission data. For example, a network coordinator can inform a node to transmit data in timeslot \#10 every three superframes starting from next superframe. Once timeslot is allocated to a node, the network coordinator also informs destination node(s) to wake up for data receiving in the allocated timeslots. The network coordinator can transmit the timeslot allocation information to source node and destination node(s) in beacon or control packet. A node follows the timeslot allocation to transmit data in CFP. The 
network coordinator records each node's timeslot assignment and the nodes allocated to each timeslot in the CFP. Any node as well as the network coordinator can initiate a timeslot allocation change at any time

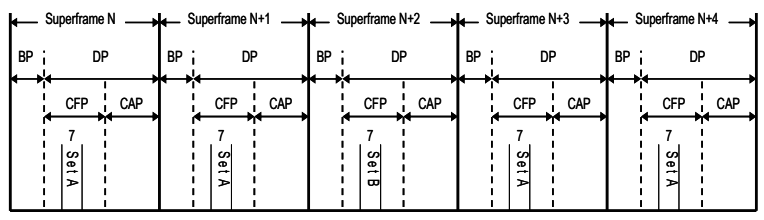

Figure 3 Set $A$ and Set $B$ share timeslot 7 for data transmission

based on node requirements or network conditions.

In a distributed wireless sensor network where a network coordinator does not exist, a node with data to transmit in CFP makes timeslot reservation by coordinating with destination node(s) so that the destination node(s) wake up to receive the data in the exact timeslot. The timeslot reservation information can be broadcasted in beacon or other control packets. The set of source node and destination node(s) that first reserves an unoccupied timeslot is called the initial set. The initial set broadcasts its data transmission pattern in the reserved timeslot. Other sets of nodes can join the initial set to share the reserved timeslot if that timeslot satisfies their data transmission needs. For example, if set A, initial set, announces that it transmits data in two of every three superframes, then a set B that transmits data in one of every three superframes can join the set A to share the timeslot with set A as shown in Figure 3.

In such a distributed wireless sensor network, a node intending to transmit data in CFP first scans for beacon or control packets to find out the occupied timeslots for sharing. If there is no occupied timeslot satisfying its needs, it then reserves the unoccupied timeslots with coordination with the destination $\operatorname{node}(\mathrm{s})$.

A set of nodes that join other set(s) to share a reserved timeslot can broadcast its data transmission pattern in that timeslot. The data transmission pattern in a reserved timeslot is propagated to the network so that no data collision is caused.

To join one or more sets and transmit data in a shared timeslot, the source node locates its turn for data transmission and informs destination node(s) so that the destination node(s) gets ready in the exact timeslot without wasting energy. A source node listens for data transmission in reserved timeslot for a sufficient number of successive superframes to determine its turn. For example, if the initial source node transmits data in a reserved timeslot every three superframes, the joining source node listens for data transmission in that timeslot for at least three successive superframes. If there is data transmission in that timeslot in each of three successive superframes, then that timeslot is not available for sharing. If the timeslot is idle in one or more superframes, the joining source node can take its turn and transmit data.

In a distributed wireless sensor network, a node can change its data transmission pattern at any time and reselect a desirable timeslot to transmit its data in CFP.

\section{Conclusion}

Wireless sensor networks have a wide range of potential applications such as target detection and tracking, environmental monitoring, industrial process monitoring, and hospital monitoring systems. Due to the short range of communication, a large number of sensor nodes are needed to perform a common task. Most importantly, sensor nodes have limited resources. Therefore, in a wireless sensor network, minimizing energy consumption and maximizing the network lifetime is a common objective. The medium access protocol must be energy efficient. The timeslot sharing medium access protocol proposed in this paper allows more sensor nodes to transmit and receive data free of contention in the guaranteed timeslots and without idle listening for receiving data. The timeslot sharing medium access protocol can increase the lifetime of a wireless sensor network by minimizing the possibility of collision. It also decreases the control packet overhead, idle listening, overemitting and overhearing. The timeslot sharing is an efficient medium access protocol for wireless sensor networks.

\section{References}

[1] Ilker Demirkol et al., "MAC Protocols for Wireless Sensor Networks: A Survey", IEEE Communications Magazine vol.44, No.4, April 2006

[2] IEEE Standard 802.15.4, "Wireless Medium Access Control (AMC) and Physical Layer (PHY) Specifications for Low-Rate Wireless Personal Area Networks (WPANs)", September 2006

[3] WiMedia Alliance, "Distributed Medium Access Control (MAC) for Wireless Networks", December 2005 
[4] IEEE Standard 802.11, "Wireless LAN Medium Access Control (MAC) and Physical Layer (PHY) Specifications, June 2007

[5] W. Ye et al, "Medium Access Control with Coordinated Adaptive Sleeping for Wireless Sensor Networks", IEEE/ACM Trans. Net., vol. 12, no. 3, June 2004

[6] A. El-Hoiydi, "Spatial TDMA and CSMA with Preamble Sampling for Low Power Ad Hoc Wireless Sensor Networks", Proc. ISCC 2002, July 2002

[7] C. C. Enz et al, "WiseNET: An Ultralow-Power Wwireless Sensor Network Solution”, IEEE Comp., vol. 37, no. 8 , Aug. 2004

[8] V. Rajendran et al, "Energy-Efficient, Collision-Free Medium Access Control for Wireless Sensor Networks", Proc. ACM SenSys 03, LA, CA, Nov. 2003 